\title{
A Influência de Tratamento Térmico Complementar sobre a Resistência ao Desgaste de Pastilha de Nitreto Cúbico de Boro
}

\author{
Guerold Bobrovnitchii, Marcello Filgueira \\ Laboratório de Materiais Avançados-LAMAV—CCT/UENF \\ Av. Alberto Lamego, 2000, pq. Califórnia, Campos dos Goytacazes, RJ. CEP: 28013-600 \\ e-mail: guerold@uenf.br, marcello@uenf.br
}

\section{RESUMO}

Os compósitos a base de nitreto cúbico de boro (cBN) atualmente se destacam na usinagem de metais e ligas de dureza elevada, podendo substituir a retífica. A efetividade de utilização dos compósitos de cNB depende, em geral, do estudo da tenacidade do inserto. A obtenção de pastilhas de cBN, realiza-se sob altas pressões até $8 \mathrm{GPa}$ e temperaturas de até $2000^{\circ} \mathrm{C}$. Em condições não hidrostáticas, as tensões internas têm valores significantes, diminuindo a vida útil da ferramenta. Para aumentar a tenacidade e a eficiência de uso foi proposto o tratamento térmico complementar em condições de vácuo sob temperaturas de até $1000^{\circ} \mathrm{C}$ e tempo de 1hora. As pastilhas de $\mathrm{cBN}$ foram sinterizadas à pressão de $7,7 \mathrm{GPa}$ e temperatura de $1600^{\circ} \mathrm{C}$. A composição da pastilha utilizada foi de $65 \%$ p de cBN e $35 \%$ p de ligante. Para determinação de nível de desgaste durante usinagem foi utilizado aço $4140 \mathrm{com}$ dureza de 56-58HRc. Comparando a efetividade de usinagem das pastilhas de $\mathrm{cBN}$ obtidas com o compósito Elbor ${ }^{\circledR}$, pôde ser notado que o tratamento térmico complementar aumentou a capacidade de usinagem da pastilha de cBN em $40 \%$.

Palavras-chave: Nitreto cúbico de boro, sinterização, desgaste, tratamento térmico.

\section{Influence of Additional Thermal Treatment on the Wear Resistance of Cubic Boron Nitride Inserts}

\section{ABSTRACT}

Cubic Boron Nitride (cBN) composites play an important role within metals and hardened alloys machining field, usually removing grinding from production processes. The effectiveness of cNB composites depends, in general, on insert toughness studies. Cubic boron nitride compacts are produced by sintering under high pressure high temperature (HPHT) conditions, up to $8 \mathrm{GPa}$ and $2000^{\circ} \mathrm{C}$. At non-hydrostatic parameters, internal stresses have significant values, decreasing tool life. In this study, the addicional thermal treatment (in vaccum, under temperatures up to $1000^{\circ} \mathrm{C}$ for 1 hour) is proposed as a way for increasing toughness and tool efficiency. The parameters used for sintering were $7.7 \mathrm{GPa}$ and $1600^{\circ} \mathrm{C}$, with samples composition of $65 \% \mathrm{w}$ of $\mathrm{cBN}$ and $35 \% \mathrm{w}$ of binder. Wear level was determined during machining of an AISI 4140 steel hardened to 56-58HRc. Comparing machining results of obtained samples and Elbor ${ }^{\circledR}$ composite, it could be noticed that the addicional thermal treatment improved cBN inserts machining capability in $40 \%$.

Keywords: Cubic boron nitride, sintering, wear, thermal treatment.

\section{INTRODUÇÃO}

No momento atual o desenvolvimento de materiais superduros (MSD), os compósitos a base de Nitreto Cúbico de Boro (cBN) tem considerável destaque [1]. Os pesquisadores de vários países desenvolvem variadas tecnologias de obtenção do material compósito que inclui micropó de cBN e ligantes (diversos metais e ligas metais de transição). Todos esses compósitos são feitos em condições de altas pressões, de 5 a $7 \mathrm{GPa}$, e temperaturas, de 1000 a $1800^{\circ} \mathrm{C}$, nos chamados dispositivos de alta pressão (DAP) [2-4]

Os materiais compósitos obtidos por este método caracterizam-se pela dispersão de tenacidade da estrutura [3] que é garantida pela ação dos seguintes fatores:

- presença de gradientes de pressão e temperatura na câmara de compressão do DAP; 
- diferença em coeficiente de expansão térmica do cBN e ligante;

- formação de novas fases com mudança de volume, como resultado da interação de componentes entre si e com cBN;

- redução rápida da pressão e temperatura.

Como a produção destes materiais compósitos realiza-se sob altas pressões, os fabricantes são dirigidos a economizar tempo do processo e diminuir o custo do DAP, e assim a redução lenta da pressão e temperatura não é implantada ainda. Essa mudança, hipoteticamente, levaria a alguma relaxação de tensões residuais no material, diminuindo assim o aparecimento de trincas que levam a diminuição da vida útil do compósito durante a usinagem [4].

Foram feitas tentativas para realizar o tratamento térmico complementar (tipo revenimento) para compósitos diretamente no dispositivo de alta pressão pela diminuição da pressão em $50-80 \%$ com diminuição simultânea da temperatura [5]. Porém, a duração daquele tratamento não é suficiente (em torno de décimos de segundo), pois a mesma sinterização ocorre durante 10-20 s e se torna impossível aumentar o tempo devido à perda de produtividade. Além do descrito, o tratamento realiza-se também em condições não equilibradas e por isso a efetividade do processo é baixa.

Foram realizadas tentativas de tratar os compósitos pelo aquecimento em atmosfera não-oxidante sem pressão sob temperaturas de $1000^{\circ} \mathrm{C}$ que não supera o valor da temperatura de estabilidade para cBN [6].

Tal tratamento pode ser realizado em fornos a vácuo ou com atmosfera protetora $\left(\mathrm{H}_{2} ; \mathrm{N}_{2}\right)$ simultaneamente para grande quantidade de amostras. Nas pesquisas de sinterização de cBN em curso na UENF, seria interessante submeter os compósitos obtidos ao tratamento térmico complementar com objetivo de melhorar a resistência ao desgaste do material policristalino obtido.

\section{MATERIAL E MÉTODOS}

A sinterização do $\mathrm{cBN}$ foi realizada sob pressão de $7,7 \mathrm{GPa}$ e temperatura de $1600^{\circ} \mathrm{C}$, utilizando ligante a base de liga $\mathrm{TiB}_{2}$-Fe. A composição da mistura reativa para sinterização foi de $65 \%$ de micropó de cBN fornecido pelo Instituto de Materiais Superduros da Ucrânia e 35\% de ligante $\left(75 \% \mathrm{TiB}_{2}+25 \% \mathrm{Fe}\right)$. As altas pressões e temperaturas durante $20 \mathrm{~s}$ foram aplicadas mediante um dispositivo de alta pressão tipo bigorna com concavidade toroidal com o diâmetro de 13,5 $\mathrm{mm}$ [7]. Foram obtidas as amostras do compósito com diâmetros de 4,0 a 4,2 $\mathrm{mm}$ e alturas de 3,5 a $4,0 \mathrm{~mm}$. As amostras previamente foram avaliadas visualmente pela ausência de trincas.

Paralelamente foram estudados os compósitos marca comercial Elbor ${ }^{\circledR}$ fornecido pelo Instituto VNIIASH, Sant Petersburgo, Rússia. As amostras fornecidas apresentaram a composição $88 \% \mathrm{cBN}+12 \%$ ligante de Si-Mg.

Para tratamento das amostras utilizou-se um forno tubular a vácuo que gerou o vácuo de $10^{-3} \mathrm{~Pa} \mathrm{e}$ temperaturas de 850 a $1000^{\circ} \mathrm{C}$. O tempo de tratamento foi de 1 hora com resfriamento de 2 horas.

A influência do tratamento sobre a efetividade de trabalho do compósito foi testada durante a usinagem do aço AISI 4140 com dureza 56-58 HRC, utilizando para tal um torno modelo ROMI TORMAX 20A. Os insertos foram fixados mecanicamente no suporte elaborado. Como parâmetros de usinagem foram utilizados: velocidade de corte de $90 \mathrm{~m} / \mathrm{s}$; avanço $0,07 \mathrm{~mm} / \mathrm{rot}$; profundidade de corte de $0,5 \mathrm{~mm}$ e tempo de processo de $5 \mathrm{~min}$. Depois do procedimento de usinagem mediu-se o desgaste na face traseira (característica da resistência da ferramenta) e a quantidade de defeitos na aresta de corte (característica da tenacidade da estrutura e capacidade de corte da ferramenta). Para cada experimento foram utilizadas oito amostras. A medição de desgaste foi realizada sob microscopia ótica, utilizando o microscópio ótico modelo NEOPHOT32.

Para estudo da influência do tratamento térmico sobre o teor de fases e estrutura do compósito obtido, utilizou-se análise de difratogramas de Raios- $\mathrm{X}$ com $\mathrm{Cu} \mathrm{k}_{\alpha}$ (Difratometro -- Marca Seifer, Modelo VRD 65) nas mesmas condições para cada amostra nos topos polidos do cilindro do compósito. ). Todas as combinações possíveis foram testadas, utilizando o software de procura por elementos e manual de busca Mineral Powder - Difraction File.

\section{RESULTADOS E DISCUSSÃO}

Como pode ser visto na Tabela 1 , o tratamento térmico sob as temperaturas de $900-950^{\circ} \mathrm{C}$ em meio inerte com duração de 15-30 min aumenta a resistência a desgaste em até $40 \%$ e diminui a quantidade de fragmentos durante a usinagem. $\mathrm{O}$ aumento da temperatura $\left(1000^{\circ} \mathrm{C}\right)$ diminui a resistência, provavelmente, devido ao aumento da temperatura da estabilidade do pó de $\mathrm{cBN}$ em condições atuais.

O tratamento do compósito Elbor ${ }^{\circledR}$ foi realizado a temperatura de $950^{\circ} \mathrm{C}$ em vácuo $\left(10^{-3} \mathrm{~Pa}\right) \mathrm{e}$ duração de 15 min. Dados da Tabela 2 mostram que o tratamento aumenta a resistência do compósito Elbor ${ }^{\circledR}$ ao desgaste e estabelece os valores experimentais. 
Tabela 1: Influência da temperatura de tratamento e sua duração sobre a resistência ao desgaste do compósito de $\mathrm{cBN}$.

\begin{tabular}{|c|c|c|c|c|}
\hline \multirow[b]{2}{*}{ Temperatura, ${ }^{\circ} \mathrm{C}$} & \multirow[t]{2}{*}{ Duração, min } & \multicolumn{2}{|c|}{ Desgaste da amostra da face traseira, $\mathrm{mm}$} & \multirow{2}{*}{$\begin{array}{l}\text { Quantidade } \\
\text { de } \\
\text { fragmentos }\end{array}$} \\
\hline & & Para cada experimento & Média & \\
\hline Material inicial & - & $\begin{array}{c}0,16 ; 0,14 ; 0,14 ; 0,13 ; \\
0,14 ; 0,12 ; 0,13 ; 0,18\end{array}$ & 0,14 & 3 \\
\hline 850 & 15 & $\begin{array}{l}0,13 ; 0,14 ; 0,17 ; 0,11 ; \\
0,10 ; 0,12 ; 0,15 ; 0,11\end{array}$ & 0,12 & 3 \\
\hline \multirow{3}{*}{900} & 15 & $\begin{array}{c}0,10 ; 0,09 ; 0,08 ; 0,09 \\
0,08 ; 0,10 ; 0,10 ; 0,11\end{array}$ & 0,10 & - \\
\hline & 30 & $\begin{array}{c}0,07 ; 0,08 ; 0,10 ; 0,10 \\
0,09 ; 0,10 ; 0,09 ; 0,10\end{array}$ & 0,09 & 1 \\
\hline & 60 & $\begin{array}{c}0,11 ; 0,08 ; 0,07 ; 0,10 \\
0,09 ; 0,09 ; 0,10 ; 0,09\end{array}$ & 0,09 & - \\
\hline 950 & 15 & $\begin{array}{c}0,10 ; 0,09 ; 0,08 ; 0,12 ; \\
0,09 ; 0,09 ; 0,10 ; 0,09\end{array}$ & 0,10 & 1 \\
\hline 1000 & 15 & $\begin{array}{c}0,10 ; 0,11 ; 0,14 ; 0,09 \\
0,12 ; 0,13 ; 0,13 ; 0,10\end{array}$ & 0,11 & 1 \\
\hline
\end{tabular}

Tabela 2: Influencia do tratamento térmico sobre a resistência do compósito Elbor ${ }^{\circledR}$.

\begin{tabular}{|c|c|c|c|}
\hline \multicolumn{3}{|c|}{ Desgaste da pastilha na face traseira, mm } \\
\hline \multicolumn{2}{|c|}{ Antes do tratamento } & Depois do tratamento \\
\hline Experimental & Média & Experimental & Média \\
\hline $\begin{array}{c}0,12 ; 0,15 ; 0,13 ; 0,11 ; \\
0,10 ; 0,11 ; 0,15\end{array}$ & 0,12 & 0,$08 ; 0,09 ; 0,09 ; 0,08 ; 0,08 ; 0,11 ;$ & 0,09 \\
\hline
\end{tabular}

A presença das seguintes fases foi confirmada (Tabela 3): cBN, $\mathrm{TiB}_{2}$ e $\mathrm{Fe}_{2} \mathrm{~B}$. A identificação de fases foi realizada para o cBN em conformidade com o trabalho []], para $\mathrm{TiB}_{2}$ e $\mathrm{Fe}_{2} \mathrm{~B}$ em conformidade com ASTM (cartões de JCPDS 2000)..Os resultados obtidos limitam-se às fichas presentes no banco de dados PDFOZ (ICDD, 1996) em software Bruker DifractPlus e ao manual.

Para todas as amostras (Tabela 4) observa-se a coincidência dos dados experimentais e padronizados: nas fases encontradas não existem orientações significativas vantajosas e ausência das macrotensões, ou seja, as tensões que não são equilibradas no volume de grão. Além disso, as distâncias interplanares, e conseqüentemente, também os parâmetros das redes das fases citadas não dependem de tratamento térmico complementar. Porém, os picos das reflexões de difração do $\mathrm{Fe}_{2} \mathrm{~B}$ em amostras iniciais são disformes, ou parte desta fase fica no estado próximo ao amorfo.

- Nas amostras iniciais o estado de fases não se diferencia fortemente (os valores das semilarguras coincidem com precisão de até 0,02 );

- As reflexões da fase de cBN não mudam a sua largura depois do tratamento;

- A largura das reflexões das fases $\mathrm{Fe}_{2} \mathrm{~B}$ e $\mathrm{TiB}_{2}$ diminui relativamente, e ao mesmo tempo a intensidade de linhas de $\mathrm{TiB}_{2}$ aumenta proporcionalmente à diminuição de sua largura, ou seja, durante o tratamento a fase $\mathrm{TiB}_{2}$ recristaliza-se parcialmente. 
Tabela 3: Intensidade relativa média $\left(\mathrm{J} / \mathrm{J}_{0}\right)$ das reflexões difratométricas das fases $\mathrm{Fe}_{2} \mathrm{~B}, \mathrm{TiB}_{2}$ e cBN nos difratogramas dos compósitos obtidos.

\begin{tabular}{|c|c|c|c|c|c|c|c|}
\hline \multirow{3}{*}{ Fase } & \multirow{3}{*}{ hkl } & \multirow{3}{*}{ Padrão } & \multicolumn{5}{|c|}{ Experimental } \\
\hline & & & \multirow{2}{*}{$\begin{array}{l}\text { Antes do } \\
\text { tratamento }\end{array}$} & \multicolumn{4}{|c|}{ Depois do tratamento } \\
\hline & & & & $900^{\circ} \mathrm{C}, 15 \mathrm{~min}$ & $900^{\circ} \mathrm{C}, 60 \mathrm{~min}$ & $950^{\circ} \mathrm{C}, 15 \mathrm{~min}$ & $\begin{array}{c}1000^{\circ} \mathrm{C}, 15 \\
\min \end{array}$ \\
\hline \multirow{10}{*}{$\mathrm{Fe}_{2} \mathrm{~B}$} & 110 & 7 & 10 & 10 & 10 & 9 & 8 \\
\hline & 200 & 15 & 10 & 14 & 12 & 15 & 15 \\
\hline & 002 & 25 & 27 & 26 & 29 & 32 & 32 \\
\hline & 121 & 100 & 100 & 100 & 100 & 100 & 100 \\
\hline & 112 & 6 & 10 & 5 & 7 & 7 & 7 \\
\hline & 220 & 6 & 10 & 5 & 7 & 7 & 7 \\
\hline & 202 & 18 & 17 & 10 & 14 & 17 & 14 \\
\hline & 113 & 18 & 17 & 10 & 14 & 17 & 14 \\
\hline & 132 & 6 & 10 & 10 & 10 & 10 & 10 \\
\hline & 400 & 6 & 10 & 10 & 10 & 10 & 10 \\
\hline \multirow{6}{*}{$\mathrm{TiB}_{2}$} & 001 & 20 & 17 & 16 & 16 & 16 & 20 \\
\hline & 100 & 60 & 44 & 43 & 48 & 46 & 49 \\
\hline & 101 & 100 & 100 & 100 & 100 & 100 & 100 \\
\hline & 002 & 13 & 14 & 15 & 14 & 17 & 15 \\
\hline & 110 & 19 & 19 & 19 & 21 & 19 & 21 \\
\hline & 102 & 15 & 17 & 17 & - & 18 & - \\
\hline \multirow{3}{*}{$\mathrm{cBN}$} & 111 & 100 & 100 & 100 & 100 & 100 & 100 \\
\hline & 200 & 6 & 8 & 6 & 8 & 8 & 8 \\
\hline & 220 & 37 & 36 & 26 & 26 & 26 & 26 \\
\hline
\end{tabular}

Tabela 4: Distâncias interplanares médias (d) para fases cBN, $\mathrm{TiB}_{2}$ e $\mathrm{Fe}_{2} \mathrm{~B}$ nos compósitos obtidos.

\begin{tabular}{|c|c|c|c|c|c|c|c|}
\hline \multirow{3}{*}{ Fase } & \multirow{3}{*}{ hkl } & \multirow{3}{*}{ d, $\AA$ (pad.) } & \multicolumn{5}{|c|}{$\mathrm{d}, \AA \AA$ experimental } \\
\hline & & & \multirow[b]{2}{*}{ Estado inicial } & \multicolumn{4}{|c|}{ Depois do tratamento } \\
\hline & & & & $\begin{array}{l}900^{\circ} \mathrm{C}, \\
15 \mathrm{~min}\end{array}$ & $\begin{array}{l}900^{\circ} \mathrm{C} \\
60 \mathrm{~min}\end{array}$ & $\begin{array}{l}950^{\circ} \mathrm{C} \\
15 \mathrm{~min}\end{array}$ & $\begin{array}{c}1000^{\circ} \mathrm{C}, \\
15 \mathrm{~min}\end{array}$ \\
\hline \multirow{3}{*}{$\mathrm{Fe}_{2} \mathrm{~B}$} & 002 & 2,12 & $2,124 \pm 0,003$ & $2,123 \pm 0,003$ & $2,124 \pm 0,003$ & $2,124 \pm 0,003$ & $2,124 \pm 0,003$ \\
\hline & 121 & 2,01 & $2,013 \pm 0,002$ & $2,014 \pm 0,002$ & $2,013 \pm 0,002$ & $2,013 \pm 0,002$ & $2,013 \pm 0,002$ \\
\hline & 202 & 1,63 & $1,635 \pm 0,001$ & $1,635 \pm 0,001$ & $1,635 \pm 0,001$ & $1,635 \pm 0,001$ & $1,635 \pm 0,001$ \\
\hline \multirow{3}{*}{$\mathrm{TiB}_{2}$} & 101 & 2,033 & $2,034 \pm 0,002$ & $2,035 \pm 0,002$ & $2,035 \pm 0,002$ & $2,035 \pm 0,002$ & $2,035 \pm 0,002$ \\
\hline & 002 & 1,618 & $1,617 \pm 0,001$ & $1,617 \pm 0,001$ & $1,618 \pm 0,001$ & $1,618 \pm 0,001$ & $1,617 \pm 0,001$ \\
\hline & 112 & 1,104 & $1,105 \pm 0,001$ & $1,105 \pm 0,001$ & $1,105 \pm 0,001$ & $1,105 \pm 0,001$ & $1,105 \pm 0,001$ \\
\hline \multirow{3}{*}{$\mathrm{cBN}$} & 111 & 2,09 & $2,085 \pm 0,002$ & $2,087 \pm 0,002$ & $2,087 \pm 0,002$ & $2,088 \pm 0,002$ & $2,086 \pm 0,002$ \\
\hline & 311 & 1,091 & $1,090 \pm 0,001$ & $1,090 \pm 0,001$ & $1,091 \pm 0,001$ & $1,090 \pm 0,001$ & $1,090 \pm 0,001$ \\
\hline & 331 & 0,831 & $0,85 \pm 0,0005$ & $0,83 \pm 0,0005$ & $0,83 \pm 0,0005$ & $0,83 \pm 0,0005$ & $0,83 \pm 0,0005$ \\
\hline
\end{tabular}

Tabela 5: Largura média de reflexões nos difratogramas.

\begin{tabular}{|c|c|c|c|c|c|c|}
\hline \multirow{3}{*}{ Fase } & \multirow{3}{*}{ hkl } & \multicolumn{5}{|c|}{ Semilargura $\mathrm{Bd}_{1}$, graus } \\
\hline & & \multirow[b]{2}{*}{$\begin{array}{c}\text { No estado } \\
\text { inicial }\end{array}$} & \multicolumn{4}{|c|}{ Após tratamento } \\
\hline & & & $\begin{array}{l}900^{\circ} \mathrm{C} \\
15 \mathrm{~min}\end{array}$ & $\begin{array}{l}900^{\circ} \mathrm{C} \\
60 \mathrm{~min} \\
\end{array}$ & $\begin{array}{l}950^{\circ} \mathrm{C} \\
15 \mathrm{~min} \\
\end{array}$ & $\begin{array}{c}1000^{\circ} \mathrm{C} \\
15 \mathrm{~min} \\
\end{array}$ \\
\hline \multirow{2}{*}{$\mathrm{Fe}_{2} \mathrm{~B}$} & 002 & $0,3 \pm 0,02$ & $0,29 \pm 0,02$ & $0,26 \pm 0,02$ & $0,29 \pm 0,02$ & $0,26 \pm 0,02$ \\
\hline & 121 & $0,3 \pm 0,02$ & $0,29 \pm 0,02$ & $0,29 \pm 0,02$ & $0,26 \pm 0,02$ & $0,27 \pm 0,02$ \\
\hline \multirow{3}{*}{$\mathrm{TiB}_{2}$} & 100 & $0,2 \pm 0,02$ & $0,21 \pm 0,02$ & $0,25 \pm 0,02$ & $0,19 \pm 0,02$ & $0,2 \pm 0,02$ \\
\hline & 101 & $0,26 \pm 0,02$ & $0,25 \pm 0,02$ & $0,27 \pm 0,02$ & $0,22 \pm 0,02$ & $0,25 \pm 0,02$ \\
\hline & 110 & $0,36 \pm 0,02$ & $0,29 \pm 0,02$ & $0,32 \pm 0,02$ & $0,29 \pm 0,02$ & $0,32 \pm 0,02$ \\
\hline \multirow{2}{*}{$\mathrm{cBN}$} & 111 & $0,22 \pm 0,02$ & $0,22 \pm 0,02$ & $0,24 \pm 0,02$ & $0,22 \pm 0,02$ & $0,22 \pm 0,02$ \\
\hline & 331 & $2,1 \pm 0,02$ & $2,1 \pm 0,02$ & $2,11 \pm 0,02$ & $2,1 \pm 0,02$ & $2,11 \pm 0,02$ \\
\hline
\end{tabular}


Considerando as semilarguras de reflexões da fase $\mathrm{TiB}_{2}$, foram feitos cálculos dos parâmetros de estrutura fina das fases por método de aproximação do perfil de reflexões difratométricas pelas funções de Gauss. Como aumento da largura instrumental foi tomada a semilargura de reflexões de $\mathrm{TiB}_{2}$ tratado termicamente a qual se manteve praticamente constante para todos os ângulos da difração e tem o valor de $0,05 \pm 0,002^{\circ}$. Os cálculos mostraram que o valor das reflexões de $\mathrm{TiB}_{2}$ em todas as amostras não superou $5.10^{-4} \AA$ e está dando a pequena contribuição no aumento das reflexões difratométricas desta fase. A razão principal em aumento das reflexões estão dados pelas microalterações (deformações) que nas amostras ficavam nos limites de $(2-2,5) \cdot 10^{-3} \AA$ e quase não dependeram de direção cristalográfica em $\mathrm{TiB}_{2}$. Depois do tratamento térmico o nível de microalterações diminuiu-se em 10-30\%.

Após tratamento térmico ocorre o aumento significativo da intensidade de reflexões difratométricas da fase $\mathrm{Fe}_{2} \mathrm{~B}$. Este aumento, provavelmente, ocorre tanto por conta de recristalização.

Nas amostras iniciais, provavelmente, tem-se pequena quantidade de Fe livre (menos de $2 \%$ ) que foi impossível identificar devido à sobreposição das linhas (110) $\alpha$-Fe com a distância interplanar $d=2,027 \AA$ sobre a linha (121) $\mathrm{Fe}_{2} \mathrm{~B}$ com $\mathrm{d}=2,01 \AA$. Neste caso, o aumento de intensidade das reflexões difratométricas pode estar ligado com a interação do cBN com o Fe livre.

\section{CONCLUSÕES}

Pelos estudos de raios-X foi determinado que durante a fabricação do material compósito no sistema cBN-TiB ${ }_{2}-\mathrm{Fe}$ em resultado da interação química do ferro com $\mathrm{cBN}$ formou-se nova fase $\mathrm{Fe}_{2} \mathrm{~B}$, ou seja, na composição do material policristalino entram as fases $\mathrm{cBN}, \mathrm{TiB}_{2}$ e $\mathrm{Fe}_{2} \mathrm{~B}$. A influência do tratamento térmico revela-se em alguma diminuição das tensões internas em cristalitos de $\mathrm{TiB}_{2}$ e também na cristalização posterior da fase $\mathrm{Fe}_{2} \mathrm{~B}$. Sobre o estado estrutural dos grãos de $\mathrm{cBN}$ o tratamento térmico não apresentou influência significativa.

Os dados da análise difratográfica satisfatoriamente explicam o aumento da qualidade do material compósito obtido a base de $\mathrm{cBN}$ sobre a influência do tratamento térmico que revela-se em aumento da resistência ao desgaste dos insertos e homogeneidade de suas propriedades.

\section{REFERÊNCIAS BIBLIOGRÁFICAS}

[1] YIN SHENG LAI-HO-YI, "High Pressure Sintering of Cubic Boron Nitride", $5^{\text {th }}$ European Symposium of Powder Metallurgy, pp. 201-211, 1978.

[2] SHIPILO, V.B., RUD, A.E., SHISHONOK, N.A., "Dependência entre Algumas Propriedades do Nitreto Cúbico de Boro e Tratamento Térmico”, Journal of Superhard Materials, n. 3, pp. 16-19, 1983.

[3] PIRKIN, N.J., ROBERTS, D.C., WILSON, W.J., “Amborite - a Remarkable New Cutting Material”, De Beers, Industrial Diamond Review, pp. 204-206, 1980

[4] FORCH, I., KUPCIK, F., "Hodnoceni Reznych Vlastrosti Polykrystalickych Desticer na Bazi Nitridu Borido", Pokroky Praskove Metalurgie, VUPM, n. 1, pp 37-46, 1986.

[5] KALININA, A.A., "Método da Obtenção do Material a Partir de Materiais Duros e Superduros e fase Cimentada", Patente da USSR, 612475, ICl B2 1/06 de 04.04.74.

[6] DRUY, M.S. "Influência de Tratamento Térmico no Vácuo Sobre Algumas Propriedades do Diamante e Elbor”, Almazy, n. 5, pp. 35-38 (em russo), 1972.

[7] KOLTCHIN, A.V., et al, "Dispositif Pour Produire um Haute Pression," Patent $N^{o}$ 2122722, Bal. NW, French, 1972.

[8] KURDIUMOV, A.V., Os Métodos de Obtenção, Propriedades e Aplicação de Nitridos, ed. Naukova Dumka, Kiev, p. 319 (em russo), 1972. 